\title{
MilliKelvin resolution in cryogenic temperature distributed fibre sensing based on coherent Rayleigh scattering
}

\author{
Xin Lu*, Marcelo A. Soto, Luc Thévenaz \\ EPFL Swiss Federal Institute of Technology, Institute of Electrical Engineering, \\ SCI STI LT, Station 11, CH-1015 Lausanne, Switzerland \\ *E-mail: xin.lu@epfl.ch
}

\begin{abstract}
The response of a distributed temperature sensor based on coherent Rayleigh scattering is experimentally studied in the temperature range from $77 \mathrm{~K}$ up to $300 \mathrm{~K}$, using fibres with standard and ORMOCER coating. A precise and absolute frequency scan is performed to obtain the best temperature resolution that turns out to be in the $\mathrm{mK}$ range. Experimental results point out that heating and cooling processes, at cryogenic temperatures, exhibit different temperature sensitivities when standard single-mode fibres are used; however, specially coated fibres exhibit better repeatability.
\end{abstract}

Keywords: Optical fibre sensors, distributed fibre sensing, Rayleigh scattering, cryogenic sensing.

\section{INTRODUCTION}

Distributed fibre sensors (DFS) based on Raman and Brillouin scattering have been commercialized and implemented for many different applications. However, they are inadequate for cryogenic temperature sensing; on the one hand, because of the low power level of the thermally-activated spontaneous Raman scattering and, on the other hand, due to the low temperature sensitivity of the Brillouin frequency shift at temperatures below $100 \mathrm{~K}^{1}$. Rayleigh-based DSF turns out to be an interesting alternative that preserves high sensitivity and temperature resolution even in harsh environmental conditions $^{2-4}$. It is particularly interesting for cryogenic applications, where extreme temperature sensitivity is requested to detect tiny fluid leakages and defects along superconductors.

Rayleigh DFS are based on the continuous natural backscattering of a coherent lightwave and the random interference originating from small stochastic fluctuations in the refractive index along the fibre. The fibre refractive index and the size of each Rayleigh scattering point are both strain and temperature dependent, and therefore, the complex amplitude of the Rayleigh scattered light at each fibre location (dependent on the local material density) changes according to the local environmental conditions ${ }^{2-4}$. The process can be locally modelled as a weak fibre Bragg grating (FBG) with random amplitude and pitch; and although Rayleigh scattering induces no frequency shift on the scattered light, the signature of the process turns out to be highly dependent on the laser frequency. The Rayleigh traces can be obtained using methods such as coherent optical frequency-domain reflectometry (C-OFDR) $)^{2,3}$ or coherent optical time-domain reflectometry $(\mathrm{C}-\mathrm{OTDR})^{4-6}$ and the jagged shape is reproducible and restorable ${ }^{6}$. By scanning the laser wavelength and comparing the obtained response with a reference, the equivalent refractive index and grating period change can be retrieved and thus information about temperature/strain variations $\mathrm{s}^{2-4}$. The technique does only provide an information about relative changes of these quantities. The high temperature/strain sensitivity could also restrict considerably the measurand range, which may require a sweeping frequency range of several tens of GHz. This requirement increases substantially the measurement time and cost of the sensing system, especially when high measurand resolution is targeted, i.e. when small frequency step are needed.

In this paper, the response of a C-OTDR based temperature sensor is measured with a highly precise and stable tuneable laser frequency to obtain an ultra-sensitive determination of any temperature change. The response is experimentally analysed at cryogenic temperatures where high sensitivity is required. The thermal sensitivity of the sensing system is over the $77 \mathrm{~K}$ to $300 \mathrm{~K}$ range, for a standard acrylate-coated single-mode fibre and a specially coated fibre with inorganic-organic hybrid materials (ORMOCERs) ${ }^{7}$. In our measurements the effect of the coating is not at all as expected, since it is not crucial in term of sensitivity, but has a clear impact on the thermo-mechanical memory of the fibre. Eventually results demonstrate that a temperature resolution of $2 \mathrm{mK}$ can be achieved over a temperature range from $100 \mathrm{~K}$ up to $300 \mathrm{~K}$.

23rd International Conference on Optical Fibre Sensors, edited by José Miguel López-Higuera,

Julian Jones, Manuel López-Amo, José Luis Santos, Proc. of SPIE Vol. 9157, 91573R

(C) 2014 SPIE - CCC code: $0277-786 X / 14 / \$ 18 \cdot$ doi: 10.1117/12.2059659 


\section{EXPERIMENT}

In C-OTDR based distributed fibre sensors, optical pulses are launched along the sensing fibre and the Rayleigh backscattering intensity is measured as function of the distance ${ }^{4-6}$. As the optical pulse propagates along the fibre, the Rayleigh backscattered light resulting from different scattering points interfere with each other, resulting in jagged shaped time-domain intensity traces.

In a C-OTDR sensor ${ }^{4}$, Rayleigh intensity traces are repeatedly acquired using different laser optical frequencies $f$, so that traces measured at a given time $t$ can be denoted as $R_{t}(z, f)$, where $f$ is scanned within a range that determines the maximum detectable temperature change. The procedure requires the use of a reference trace $R_{r}\left(z, f^{\prime}\right)$, which is then cross-correlated in frequency with the actual Rayleigh measurement $R_{t}(z, f)$. The cross-correlation $X \operatorname{corr}\left(z_{0}, f-f^{\prime}\right)=$ $R_{t}\left(z_{0}, f\right) * R_{r}\left(z_{0}, f^{\prime}\right)$ gives the information of the frequency shift $\Delta f=f-f^{\prime}$ induced by temperature or strain changes (at a given position $z_{0}$ ).

The purpose of this paper is to investigate the response of Rayleigh distributed sensors in a wide temperature range; however, a large temperature change $\Delta T$ might induce a frequency shift $\Delta f$ that is beyond the total scanning range of a given C-OTDR sensor, leading to the indetermination of $\Delta T$. A simple solution to extend the temperature sensing range of C-OTDR sensors is to cross-correlate the measurement $R_{t_{i-1}}(z, f)$ with the following one $R_{t_{i}}(z, f)$, both obtained within a small scanning frequency range $\Delta f_{i}$, so that only low frequency scanning is required. This way, an equivalent wide frequency scan $\Delta f$ can be obtained using only incremental measurements, so that the total scanned frequency shift can be expressed as $\Delta f=\sum_{i=1}^{N} \Delta f_{i}, N$ being the number of measurements.

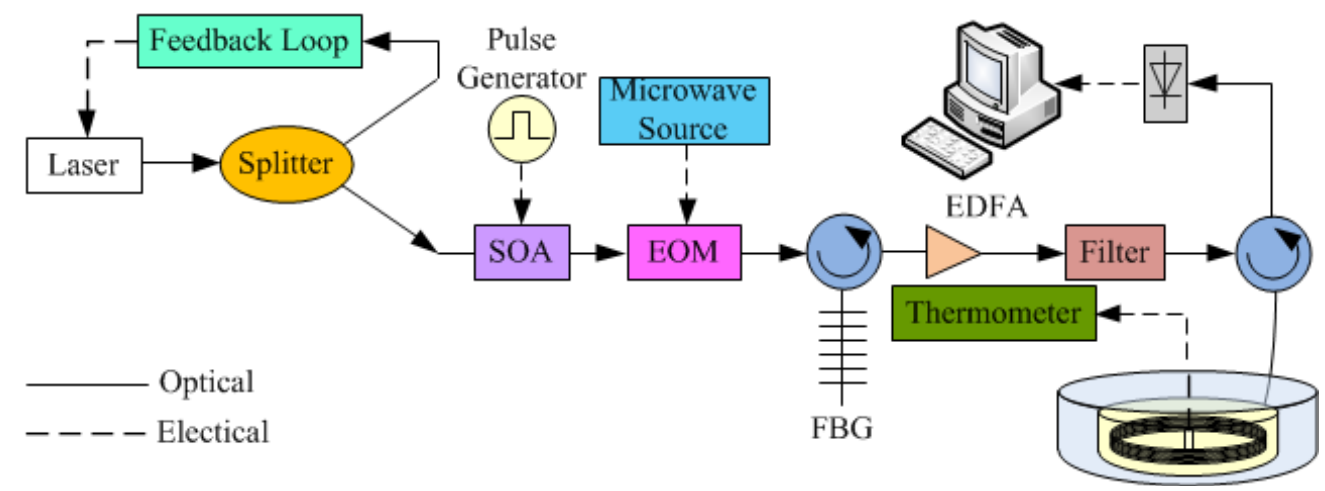

Figure 1. Implemented C-OTDR distributed sensor.

The experiment setup is sketched in Fig. 1. The continuous-wave light from a distributed-feedback laser is divided into two paths through an optical splitter. The upper path in the figure is connected to a feedback loop that locks the laser frequency on one of the absorption lines of a hollow-core fibre gas cell filled with acetylene, securing a stable absolute laser frequency. In the lower path, a semiconductor optical amplifier (SOA) is used to generate pulses with high extinction ratio ${ }^{5}$ and $17 \mathrm{~ns}$ width, corresponding to a spatial resolution of $1.7 \mathrm{~m}$. The optical frequency of the pulses is scanned using an electro-optic modulator (EOM) driven by a frequency microwave source. An FBG is employed to select one of the two sidebands generated by the EOM. Then, optical pulses are amplified by an Erbium-doped fibre amplifier (EDFA) and a filter is used to suppress the amplified spontaneous emission (ASE) noise. Pulses are launched into the fibre under test (FUT) and the Rayleigh signal is collected by a $125 \mathrm{MHz}$ photodetector.

In order to reduce temperature gradients along the FUT, short fibre segments are loosely coiled inside a copper box with a diameter of $9 \mathrm{~cm}$. The sensing fibre consists of 2 appended distinct fibres: a 7 metre standard single-mode fibre followed by a 4-metre special fibre with ORMOCER coating ${ }^{5}$. This allows the simultaneous comparative analysis of two different fibres.

For each measurement, 1000 Rayleigh traces are averaged at each scanned frequency; the frequency step is set to $10 \mathrm{MHz}$ and the scanning range to $400 \mathrm{MHz}$. In order to precisely retrieve the peak cross-correlation frequency and to best interpolate the effect of the discrete spectral scan, the spectrum at each fibre location is fitted to a quadratic curve.

For measurements at room temperature, the copper box is placed in a thermal bath filled with water. The large specific heat capacity of water and the thermal buffering offered by the metallic box provides an excellent uniform temperature distribution along the entire FUT. A Pt-1000 temperature probe, placed in the middle of the metallic box, is used for monitoring and calibration. For cryogenic temperature measurements, the copper box is placed in a liquid nitrogen 
container. A direct contact of the box with the liquid allows a precise and stable temperature measurement at $\sim 77 \mathrm{~K}$; however, to vary the temperature, the box is positioned inside a second tank, which also acts as a thermal screen that maintains the sensing fibres at a temperature higher than the liquid Nitrogen. Measurements at different temperatures have been possible by controlling the speed of evaporation of the liquid.

\section{RESULTS AND DISCUSSION}

The result of the cross-correlation between two successive measurements is presented in Fig. 2(a). The inset shows the cross-correlation at a given position, peaking at a definite frequency shift determined by a quadratic fitting. The estimated frequency shift as a function of the distance is also illustrated in the figure. The high temperature sensitivity of the method makes the cross-correlation peak shifts in frequency even under very small temperature changes. In the case depicted in Fig. 2(a), the fibre temperature changed by $\sim 2 \mathrm{mK}$, inducing a frequency variation of about $25 \mathrm{MHz}$ in both sensing fibres. The standard deviation obtained along the fibres is between 2 and $4 \mathrm{MHz}$, which corresponds to a temperature accuracy between 2 and $4 \mathrm{mK}$ in ambient conditions. This frequency uncertainty is mainly given by the noise characteristics of the sensing system and the frequency scanning step, so that it is independent on the fibre temperature.
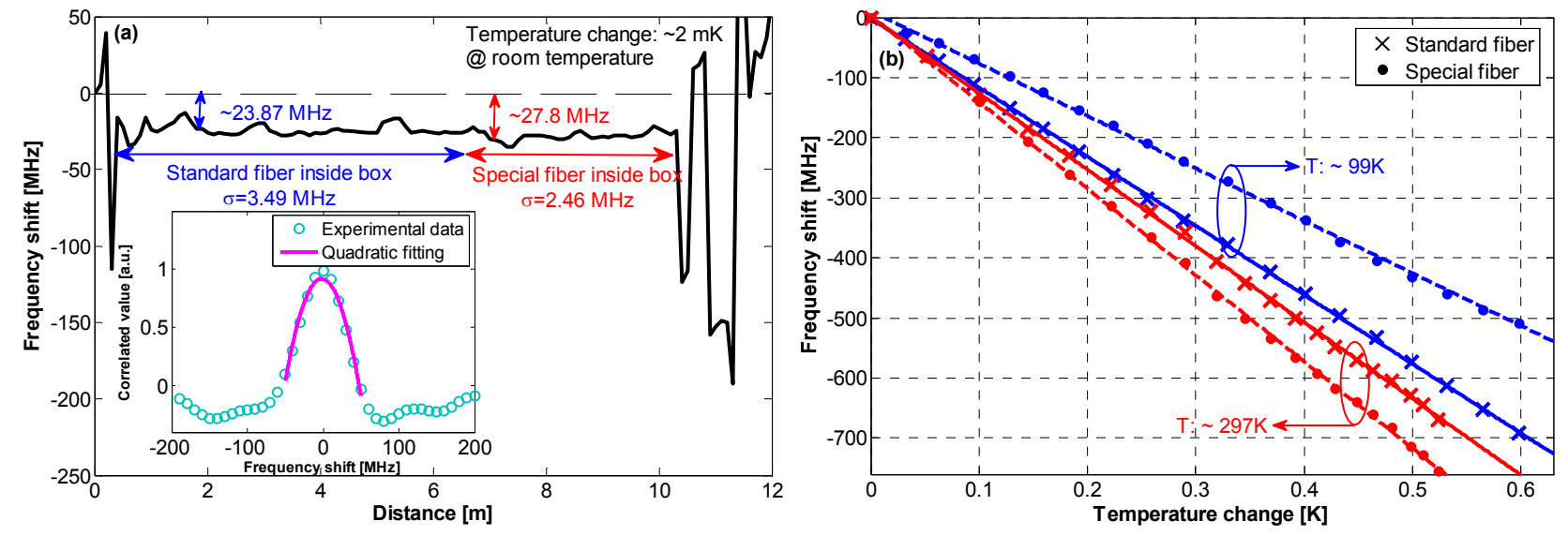

Figure 2. (a) Frequency shift vs the distance, inset: quandratic fitting of the correlated peak; (b) Frequency shift at $297 \mathrm{~K}$ and $99 \mathrm{~K}$.

The response of the C-OTDR sensor is measured at different temperatures, from $77 \mathrm{~K}$ up to $300 \mathrm{~K}$. Fig. 2(b) shows the frequency shifts obtained under cryogenic and ambient conditions. Different sensitivities (represented by the slopes in the plots) can be observed for the different cases, as well as for both types of fibre. The sensitivity decrease under cryogenic environment conditions is usually explained by the reduced thermal expansion of silica at low temperatures.
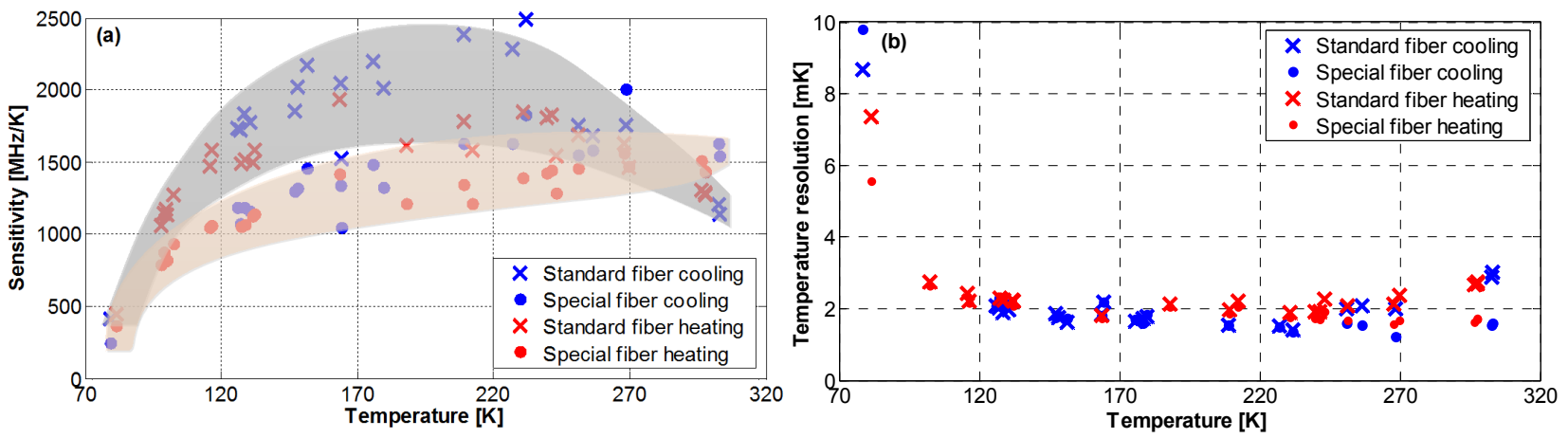

Figure 3. Temperature sensitivity (a) and resolution (b) of the implemented C-OTDR sensor at different temperatures.

Fig. 3(a) summarises the temperature sensitivity measured when negative and positive temperature variations are applied along the two sensing fibres. The figure depicts a grey-shaded region representing the range within which most of the measured points have been obtained for the standard fibre; while the pink-shaded region corresponds to the range for the special fibre with ORMOCER coating. Results confirm the high temperature sensitivity of Rayleigh scattering over the 
entire 77-300 K temperature range. In particular, it is possible to observe that, for the standard acrylate-coated fibre, the highest temperature sensitivity is reached around $210 \mathrm{~K}$. But this fibre shows clear discrepancies between positive and negative thermal gradients. This kind of thermal hysteresis is similar to the one reported for standard FBG sensors ${ }^{8}$ and might be caused by the refractive index change induced by the residual stress reliefs. Comparatively, the special fibre presents better repeatability in both thermal change directions. This could be because the hybrid polymers inside the ORMOCER coating greatly stabilize the mechanical and thermal response of the fibre ${ }^{9}$. It must be pointed out that the ORMOCER coating does not bring clear benefit in term of sensitivity over this temperature range, since even a reduced response is observed under $200 \mathrm{~K}$. But it must be mentioned that this fibre presents a high core doping concentration and this may also have a significant impact on the thermal response of the refractive index.

Based on the measured frequency accuracy $\sigma_{v}(z)$ and on the temperature sensitivity reported in Fig. 3(a), the temperature resolution can be easily deduced. Fig. 3(b) shows this resolution as measured during heating and cooling processes over the $77-300 \mathrm{~K}$ range, for both types of fibre. The worst resolution is observed to be $\sim 9 \mathrm{mK}$ around liquid Nitrogen temperature; however, a remarkable temperature resolution of $\sim 2 \mathrm{mK}$ is obtained between $150 \mathrm{~K}$ and $300 \mathrm{~K}$. This resolution is indicative only, since it is highly dependent on the signal-to-noise ratio of the signal that can be worse at long distances, but can be improved by longer averaging and spatial resolution. It can also be improved using a smaller frequency scanning step ${ }^{10}$.

\section{CONCLUSION}

A high temperature sensitivity and resolution is experimentally evaluated for a cryogenic temperature sensor based on C-OTDR, revealing a potential for broadening the application domain of DFS. A better repeatability during heating and cooling processes is observed for the FUT with ORMOCER coating. But further investigation is still required to better understand the impact of fibre parameters on the performance of Rayleigh based DFS at cryogenic condition.

This work was performed within the framework of ITER-EPFL Task Agreement (Project IO/CFT/11/5840/RTS). The views and opinions expressed herein do not necessarily reflect those of the ITER organization. The authors warmly thank Dr Kay Schuster from IPHT, Jena, for providing the ORMOCER-coated fibre sample.

\section{REFERENCES}

[1] Fellay, A., Thévenaz, L., Garcia, J.P., Facchini, M., Scandale, W. and Robert, P., "Brillouin-based temperature sensing in optical fibres down to $1 \mathrm{~K}$," Optical Fiber Sensors Conference Technical Digest, 2002. OFS 2002, 15th, 301-304 (2002)

[2] Boyd, C., Lally, E., Horrell, E. and Dickerson, B., "Verifying cryogenic cooling of superconducting cables using optical fiber," Future of Instrumentation International Workshop 2012 (FIIW), 1-4 (2012)

[3] Sang, A., Froggatt, M., Gifford, D., Kreger, S. and Dickerson, B., "One centimeter spatial resolution temperature measurements in a nuclear reactor using Rayleigh scatter in optical fiber," IEEE Sensors J., 8(7), 1375-1380 (2008).

[4] Koyamada, Y., Imahama, M., Kubota, K. and Hogari, K., "Fiber-optical distributed strain and temperature sensing with very high measurand resolution over long range using coherent OTDR," J. Lightwave Technol. 27(9), 11421145 (2009).

[5] Martins, H., Martin-Lopez, S., Corredera, P., Filograno, M., Frazao, O. and Gonzalez-Herraez, M., "Coherent noise reduction in high visibility phase-sensitive optical time domain reflectometer for distributed sensing of ultrasonic waves," J. Lightwave Technol. 31(23), 3631-3637 (2013).

[6] Imahama, M., Koyamada, Y. and Hogari, K., "Restorability of Rayleigh backscatter traces measured by coherent OTDR with precisely frequency- controlled light source," IEICE Trans. Commun., E91-B(4), 1243-1246(2008).

[7] Habisreuther, T., Hailemichael, E., Ecke, W., Latka, I., Schroder, K., Chojetzki, C., Schuster, K., Rothhardt, M. and Willsch, R., "ORMOCER coated fiber-optic Bragg grating sensors at cryogenic temperatures," IEEE Sensors J, 12(1), 13-16 (2012).

[8] Douay, M., Fertein, E., Xie, W. X., Bernage, P., Niay, P., Bayon, J. F. and Georges, T., "Thermal hysteresis of Bragg wavelengths of intra-core fiber gratings," Photon. Technol. Lett. 5(11), 1331-1334 (1993).

[9] Schuster, K., Kobelke, J., Rose, K., Helbig, M., Zoheidi, M. and Heinze, A., "Innovative fiber coating systems based on organic modified ceramics," Proc. SPIE 7598, Optical Components and Materials VII, 75980H (2010).

[10] Soto, M. and Thévenaz, L., "Modeling and evaluating the performance of Brillouin distributed optical fiber sensors," Opt. Express 21(25), 31347-31366 (2013) 